\title{
NOUVELLe
}

\section{Un vaccin ciblant les cytokines IL-4 et IL- 13 protège contre l'asthme allergique chez la souris}

Eva Conde ${ }^{1,2}$, Vincent Serra ${ }^{2}$, Pierre Bruhns ${ }^{1}$, Laurent L. Reber ${ }^{3}$
${ }^{1}$ Unité des anticorps en thérapie et pathologie, Institut Pasteur, Inserm UMR1222, 75015 Paris, France.

${ }^{2}$ Neovacs SA, Paris, France.

${ }^{3}$ Institut toulousain des maladies infectieuses et inflammatoires (Infinity), Inserm UMR1291, CNRS UMR5051, Université Paul Sabatier Toulouse III, 31024 Toulouse, France. laurent.reber@inserm.fr
> L'asthme est une maladie pulmonaire chronique de très forte prévalence, touchant quatre millions de personnes en France et plus de 300 millions dans le monde [1]. Bien que l'asthme soit une maladie hétérogène regroupant des phénotypes variés, environ la moitié des sujets asthmatiques développent une inflammation de type 2, caractérisée par des taux élevés d'anticorps de classe Ig(immunoglobuline) $\varepsilon$, de cytokines de type Th2, comme l'interleukine 4 (IL-4) et IL-13, et par la présence de granulocytes éosinophiles dans les voies aériennes $[1,2]$.

Les corticoïdes administrés par inhalation sont le traitement de base dans l'asthme. Cependant, dans les cas d'asthme sévère, cette corticothérapie locale ne suffit pas à réduire les symptômes. Au cours des dernières années, plusieurs anticorps monoclonaux ciblant les $\lg \varepsilon$, les cytokines Th2 ou leurs récepteurs ont été utilisés pour traiter l'asthme sévère [3], mais l'utilisation de ces anticorps est fortement limitée par leur coût, et la nécessité d'effectuer des réinjections fréquentes (toutes les deux à quatre semaines) ${ }^{1}$. Pour pallier cet inconvénient, nous avons développé, dans un partenariat entre l'Inserm, I'Institut Pasteur et l'entreprise NEOVACS, des vaccins conjugués, appelés kinoïdes [11] $(\rightarrow)$ permettant d'induire une production endogène d'anticorps neutralisants dirigés contre l'IL-4 et I'IL-13. Ces kinoïdes

$(\rightarrow)$ Voir la Synthèse de A. Bensussan et al., $m / s n^{\circ} 3$, mars 2008, page 306

1 Le benralizumab (Fasenra ${ }^{\circledR}$ ), un anticorps non fucosylé dirigé contre la chaîne alpha du récepteur de l'IL-5, déplète les granulocytes éosinophiles et est indiqué dans l'asthme sévère avec une injection par voie sous-cutanée seulement tous les deux mois. sont produits en couplant I'IL-4 et l'IL-13 recombinantes à une protéine porteuse appelée $\mathrm{CRM}_{197}$ (cross-reacting material 197), une version mutante non-toxique de la toxine diphtérique déjà utilisée dans un certain nombre de vaccins conjugués [4] (Figure 1).

Nous avons d'abord produit des kinoïdes à partir de I'IL-4 et de I'IL-13 murines, afin d'obtenir une preuve de concept de l'efficacité de ces candidats vaccins chez la souris. Une vaccination de souris $\mathrm{BALB} / \mathrm{c}$ par voie intramusculaire avec une émulsion de ces kinoïdes en pré- sence d'un adjuvant à base de squalène (SWE) [5] a induit une forte production d'anticorps polyclonaux de type IgG dirigés contre I'IL-4 et I'IL-13. Ces anticorps ont une forte capacité neutralisante, puisqu'ils étaient capables d'inhiber l'activité des deux interleukines dans des tests in vitro. Cette réponse anticorps était encore observable chez plus de $60 \%$ des souris un an après la primovaccination : elle est donc durable.

Nous avons ensuite évalué l'efficacité des kinoïdes IL-4 et IL-13 dans un modèle murin d'asthme chronique induit

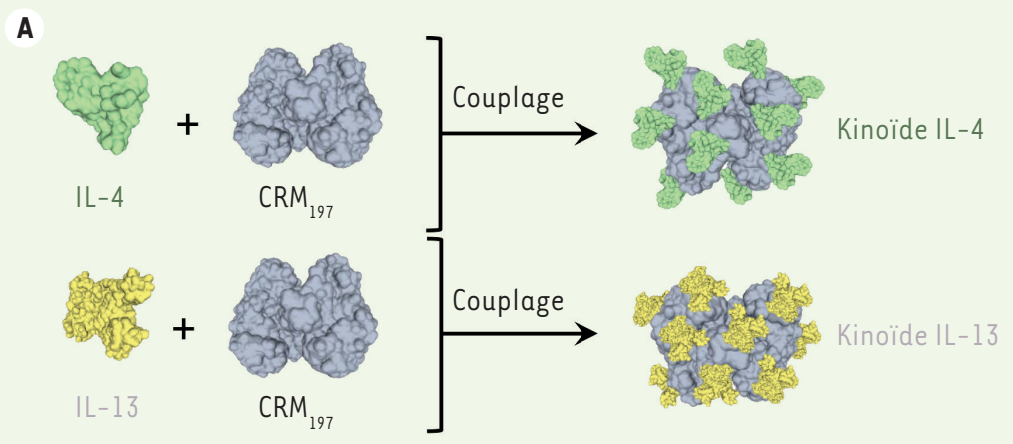

B
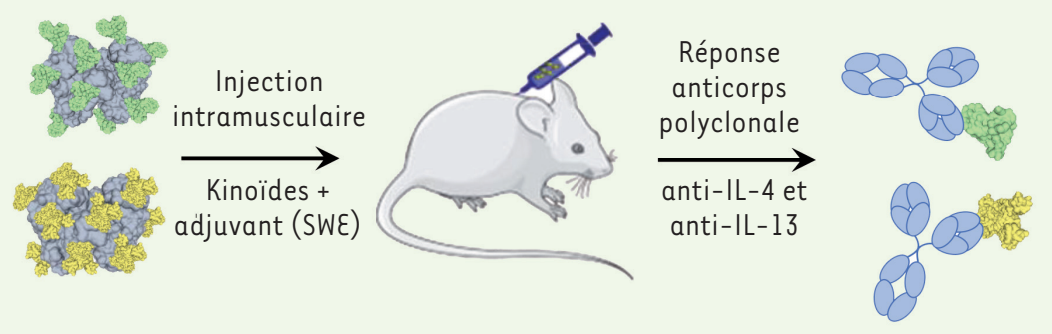

Figure 1. Vaccin kinoïde IL-4/IL-13. A. Les kinoïdes IL-4 et IL-13 sont synthétisés par couplage chimique de I'IL-4 ou de l'IL-13 recombinante avec la protéine porteuse CRM 197 . B. Chez la souris, l'injection intramusculaire de kinoïdes IL-4 et IL-13 en émulsion avec un adjuvant à base de squalène (SWE) induit une réponse anticorps polyclonale neutralisante contre l'IL-4 et l'IL-13 endogène. 


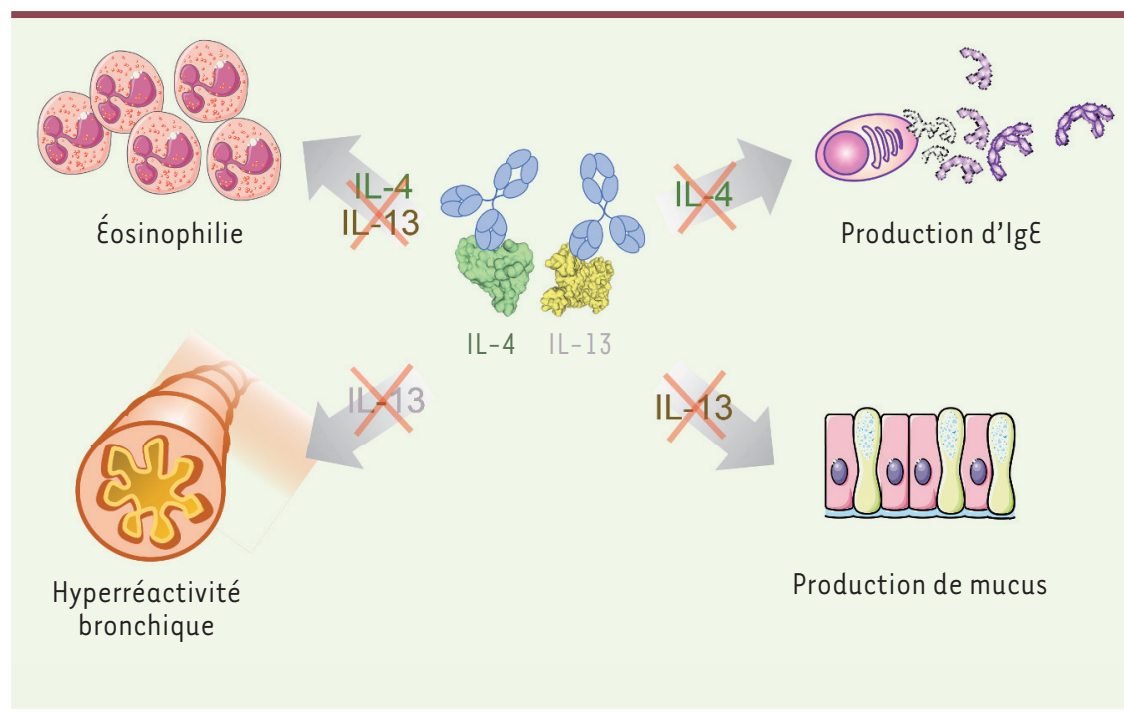

par des administrations intranasales répétées d'extraits d'acariens (les acariens sont à l'origine des allergènes majeurs impliqués dans l'asthme). Ce modèle permet de reproduire les principales caractéristiques de l'asthme de type 2 : production d'un fort taux d'lge, hyperréactivité bronchique, inflammation impliquant des granulocytes éosinophiles et hypersécrétion de mucus. Une vaccination prophylactique de ces souris avec les kinoïdes IL-4 ou IL-13 a entraîné une réduction marquée des taux d'lgk, avec un effet du vaccin anti-IL-4 supérieur à celui du vaccin anti-IL-13. L'hyperréactivité bronchique et la production de mucus ont également diminué considérablement chez les souris vaccinées, avec cette fois, un effet beaucoup plus marqué pour le vaccin anti-IL-13 que pour le vaccin anti-IL-4. Finalement, le recrutement de granulocytes éosinophiles dans les voies aériennes a également été réduit chez les souris ayant reçu l'un ou l'autre vaccin, et davantage encore chez celles vaccinées avec les deux kinoïdes de manière combinée (Figure 2).

Ces résultats ont mis en évidence le fait que I'IL-4 et I'IL-13 exercent certaines activités non redondantes dans l'asthme, ce qui a également été observé chez l'homme. En effet, l'anticorps thérapeutique dupilumab (qui cible de manière combinée les voies
IL-4 et IL-13 en bloquant la sous-unité IL-4R $\alpha$ commune aux récepteurs de ces deux cytokines) a une efficacité clinique dans l'asthme supérieure à celle d'anticorps thérapeutiques ciblant soit I'IL-4, soit I'IL-13 [6-8]. Nos résultats précliniques ont également démontré le potentiel thérapeutique du vaccin combiné ciblant à la fois I'IL-4 et I'IL13, puisque celui-ci a également été capable de réduire les taux d'lgE, le nombre de granulocytes éosinophiles, l'hyperréactivité bronchique et la production de mucus dans un protocole de vaccination thérapeutique.

Forts de ces résultats chez la souris, nous avons alors développé des kinoïdes à partir de I'IL-4 et de I'IL-13 humaines. Afin de pouvoir tester ces candidats vaccins in vivo, nous avons utilisé un nouveau modèle de souris transgéniques exprimant les formes humaines d'IL-4, d'IL-13 et de I'IL-4R $\alpha$ à la place des formes murines de ces molécules. Dans ce modèle murin «humanisé », une vaccination combinée avec les kinoïdes IL-4/ IL-13 humaines a permis d'induire la production d'anticorps neutralisants contre les deux cytokines humaines. De plus, cette vaccination a réduit de manière durable les taux d'IgE circulantes, confirmant l'efficacité de ce vaccin.

Ces résultats précliniques encourageants ont été publiés récemment [9], et doivent maintenant être confirmés
Figure 2. Effet des kinoïdes IL-4 et IL-13 dans un modèle murin d'asthme. Une vaccination avec les kinoïdes IL-4 et IL-13 réduit fortement les taux d'Ige dans un modèle murin d'asthme aux acariens, avec un effet plus prononcé pour le vaccin anti-IL-4 que pour le vaccin anti-IL-13. L'hyperréactivité bronchique et la production de mucus sont également considérablement diminuées dans les souris vaccinées, avec, dans ce cas, des effets beaucoup plus marqués pour le kinoïde IL-13 que pour le kinoïde IL-4. Le recrutement de granulocytes éosinophiles dans les voies aériennes est inhibé par l'un et l'autre kinoïde, et l'efficacité est accrue quand ces deux kinoïdes sont administrés de manière combinée.

par des essais cliniques chez l'homme. Un kinoïde ciblant une autre cytokine, l'interféron alpha (IFN- $\alpha$ ), a récemment été testé par NEOVACS dans un essai clinique de Phase Ilb chez 185 adultes atteints de lupus érythémateux aigu disséminé. Ce kinoïde a induit une réponse neutralisante contre I'IFN- $\alpha$ chez $91 \%$ des patients traités, avec une bonne tolérance du vaccin [10]. Bien qu'il reste à déterminer l'efficacité et la durée de la réponse anticorps induite par les kinoïdes IL-4/IL-13 par des essais cliniques, celles-ci pourraient être similaires aux réponses observées avec le kinoïde IFN- $\alpha$. Outre l'asthme, nous pensons que d'autres maladies allergiques, telles que la dermatite atopique, la rhinosinusite chronique avec polypes nasaux, ou l'allergie alimentaire, dans lesquelles I'IL-4 et I'IL-13 jouent également un rôle majeur, pourraient, dans leurs formes sévères, bénéficier également de la vaccination par les kinoïdes IL-4/IL-13. $\diamond$

A vaccine targeting the cytokines $\mathrm{IL}-4$ and IL- 13 protects against allergic asthma in mice

\section{LIENS D'INTÉRÊT}

Eva Conde, Pierre Bruhns, Vincent Serra et Laurent Reber sont co-inventeurs du brevet W02019228674 «produit immunogène comprenant I'IL-4 et/ou l'IL-13 pour le traitement de troubles associés à l'expression ou à l'activité aberrante de I'IL-4 et/ou de I'IL-13 ». Vincent 
Serra est CSO/COO de NEOVACS. Eva Conde est une ancienne employée de NEOVACS. Pierre Bruhns et Laurent Reber ont exercé une activité de consultance auprès de NEOVACS.

\section{RÉFÉRENCES}

1. Fahy JV. Type 2 inflammation in asthma: present in most, absent in many. Nat Rev Immunol 2015 ; 15 : 57-65.

2. Lambrecht BN, Hammad H, Fahy JV. The cytokines of asthma. Immunity $2019 ; 50: 975-91$.

3. Barnes PJ. Targeting cytokines to treat asthma and chronic obstructive pulmonary disease. Nat Rev Immunol 2018; $18: 454-66$.
4. Malito $\varepsilon$, Bursulaya B, Chen C, et al. Structural basis for lack of toxicity of the diphtheria toxin mutant CRM197. Proc Natl Acad Sci USA 2012 ; 109 : 5229-34.

5. Ventura R, Brunner L, Heriyanto B, et al. Technology transfer of an oil-in-water vaccine-adjuvant for strengthening pandemic influenza preparedness in Indonesia. Vaccine 2013 ; 31 : 1641-5.

6. Bagnasco D, Ferrando M, Varricchi G, et al. A critical evaluation of anti-IL-13 and anti-IL-4 strategies in severe asthma. Int Arch Allergy Immunol 2016; 170 : $122-31$.

7. Hanania NA, Korenblat P, Chapman KR, et al. Efficacy and safety of lebrikizumab in patients with uncontrolled asthma (LAVOLTA I and LAVOLTA II): replicate, phase 3, randomised, double-blind, placebo-controlled trials. Lancet Respir Med 2016; 4 : 781-96.
8. Wenzel S, Ford L, Pearlman D, et al. Dupilumab in persistent asthma with elevated eosinophil levels. $N$ Engl J Med $2013 ; 368: 2455-66$.

9. Conde $\varepsilon$, Bertrand R, Balbino B, et al. Dual vaccination against IL-4 and IL- 13 protects against chronic allergic asthma in mice. Nat Commun 2021 ; $12: 2574$.

10. Houssiau FA, Thanou A, Mazur M, et al. IFN-alpha kinoid in systemic lupus erythematosus: results from a phase IIb, randomised, placebo-controlled study. Ann Rheum Dis 2020 ; 79 : 347-55.

11. Bensussan A, Bizzini B, Pouletty P, et al. Les kinoïdes: une nouvelle génération de vaccins thérapeutiques. Med Sci (Paris) 2008 ; 24 : 306-13. 